\title{
A Simple and Effective Transplantation Device for Zebrafish Embryos
}

\author{
Gary H. Soh ${ }^{1}$, Anna C. Kögler ${ }^{1}$, Patrick Müller ${ }^{1,2}$ \\ ${ }^{1}$ Systems Biology of Development Group, Friedrich Miescher Laboratory of the Max Planck Society ${ }^{2}$ University of Konstanz
}

\section{Corresponding Author}

Patrick Müller

patrick.mueller@tuebingen.mpg.de

\section{Citation}

Soh, G.H., Kögler, A.C., Müller, P. A Simple and Effective Transplantation Device for Zebrafish Embryos. J. Vis. Exp. (174), e62767, doi:10.3791/62767 (2021).

\section{Date Published}

August 2, 2021

\section{DOI}

$10.3791 / 62767$

URL

jove.com/video/62767

\section{Abstract}

Classical embryological manipulations, such as removing cells and transplanting cells within or between embryos, are powerful techniques to study complex developmental processes. Zebrafish embryos are ideally suited for these manipulations since they are easily accessible, relatively large in size, and transparent. However, previously developed devices for cell removal and transplantation are cumbersome to use or expensive to purchase. In contrast, the transplantation device presented here is economical, easy to assemble, and simple to use. In this protocol, we first introduce the handling of the transplantation device as well as its assembly from commercially and widely available parts. We then present three applications for its use: generation of ectopic clones to study signal dispersal from localized sources, extirpation of cells to produce size-reduced embryos, and germline transplantation to generate maternalzygotic mutants. Finally, we show that the tool can also be used for embryological manipulations in other species such as the Japanese rice fish medaka.

\section{Introduction}

From the classical experiments of Mangold and Spemann that demonstrated the existence of an organizer instructing the formation of an embryonic axis ${ }^{1}$, transplantation of cells between embryos has become an established technique for studying embryonic development ${ }^{2,3,4,5,6,7,8,9,10}$. A commonly used setup for transplantation consists of a micrometer drive-controlled gas-tight syringe connected to a micropipette holder through flexible tubing and a reservoir filled with mineral oil ${ }^{12,13}$. In this setup, the plunger of the syringe is moved through a screw. The pressure generated in this manner is transferred to the micropipette and used to draw cells out from one embryo and deposit them into another. However, this hydraulically operated device consists of many parts and is laborious to assemble from scratch. Similar devices can also be purchased as a complete working set, usually sold as Manual Microinjectors, and these commercial versions typically cost more than 1500 US\$. In both the home-made and the commercial version, the micropipette for embryo manipulation is separated from the pressure-generating device (the gas-tight syringe) through oil-filled tubing. The manipulation of the micropipette and the movement of the plunger, therefore, have to be operated 
separately with different hands, reducing the throughput and utility. Furthermore, the devices are cumbersome to prepare for transplantation since the tubing needs to be carefully filled with oil while avoiding the formation of bubbles. Here, we describe an alternative pneumatically operated device for cell removal and transplantation that is inexpensive, easy to assemble, and simple to use.

The device presented here comprises a $25 \mu \mathrm{L}$ gas-tight syringe fitted with a micropipette holder and costs less than 80 US $\$$ altogether. The device is easily assembled by inserting the micropipette holder into the syringe via Luer lock fitting (Figure 1A). The device is then directly mounted onto a micromanipulator, allowing the user to control both its position and the suction with a single hand directly at the micromanipulator. This conveniently leaves the other hand free to stabilize and move the transplantation dish containing donor and host embryos. The device works by direct suction with air and does not need to be filled with mineral oil. Due to the attractive forces between the water and the walls of the glass needle, a large movement in the plunger of the syringe is translated into a smaller movement in the water level within the needle, as long as the water level is in the tapered end of the glass needle. This allows precise control over the number of aspirated cells and the location of their insertion.

To demonstrate the utility of this device, we present three applications in zebrafish (Danio rerio) embryos. First, we show how to generate localized sources of secreted signaling molecules, which can be used to study gradient formation ${ }^{2,4,6}$. Here, donor embryos are injected with mRNA encoding a fluorescently labeled signaling molecule. The fluorescence-labeled donor cells are then transplanted to wild-type host embryos where the formation of a signal gradient can be imaged and analyzed. Second, we describe how the device can be used to remove cells by extirpation in order to generate size-reduced embryos ${ }^{5,13}$. Finally, we show how to robustly produce maternal-zygotic mutants by transplanting cells carrying a primordial germ cell reporter into host embryos in which the germ line had been ablated ${ }^{6,10}$. In the future, the transplantation device described here can be easily adapted to other embryological manipulations requiring the removal or transplantation of cells.

\section{Protocol}

\section{Assembling and using the transplantation device}

1. Assembling the transplantation device.

1. Connect the Luer tip $25 \mu \mathrm{L}$ gas-tight syringe and a micropipette holder with Luer lock fitting to assemble the transplantation device (Figure 1A).

NOTE: Wetting the Luer tip with a thin film of water can help to enhance the connection by holding the parts together via water adhesion and cohesion.

2. Mount the device directly onto a manual micromanipulator (Figure 1B). The device can be controlled with the dominant hand alone, freeing up the other hand for additional tasks.

2. Preparing the transplantation needle.

1. Produce a transplantation needle by pulling a glass capillary pipette (without filament) with a micropipette puller.

2. Break the tip of the needle as smoothly as possible, as sharp edges increase the chance of scratching the yolk while transplanting, which is fatal to the embryo. 
NOTE: The tip can be easily broken with a straight-edge razor blade under a stereomicroscope. However, using a microforge allows to generate a precise opening of the desired size. For ectopic source generation, an outer diameter of approximately $50-60 \mu \mathrm{m}$ is appropriate. For extirpations and germline transplantation, the outer diameter of the needle should measure approximately $80-90 \mu \mathrm{m}$ to increase the number of transplanted cells.

3. Insert the ready-to-use needle into the transplantation device (Figure 1A).

3. Using the transplantation device.

1. Place the micromanipulator with the transplantation device next to a stereomicroscope (Figure 1B).

2. Remove the plunger and lower the transplantation needle into the transplantation dish filled with Ringer's solution (see step 2.2.1) at a $45^{\circ}$ angle until the tip of the needle is immersed (Figure 1B). Water will rush up the needle due to capillary action.

3. Insert the plunger approximately halfway to flush out the Ringer's solutions, leaving only a small volume of water in the thin, tapered part of the needle (Figure 1C).

NOTE: This is the neutral position, and the water level should be stable there for a while (>20 $\mathrm{min}$ ). If the water level is unstable, the air is leaking; reconnect the Luer lock fitting or replace the syringe.

4. When using a transplantation needle for the first time, coat its inside by drawing up yolk from a sacrificed embryo and then expelling the yolk material completely. The coating will help to reduce the adherence of cells to the glass during subsequent procedures.

5. Gently position the donor embryo with the help of the needle and then position the needle opening orthogonal to the embryo's surface (Figure 1D).

NOTE: The position will be different for different assays. For ectopic signaling molecule source generation and cell extirpations, this will be the top of the animal pole (Figure 1E); for germline transplantation, this will be the margin (Figure 1D,F).

6. Slowly and carefully pull up the plunger to draw the cells into the needle.

NOTE: If the cells are taken up too quickly, they can be damaged. If done correctly, the cells should come out as a cylindrical column. Avoid taking up yolk into the needle, as the transplanted yolk is toxic for the host embryo.

7. Stop the suction by gently pushing the plunger down slightly once the desired number of cells is drawn in. Remove the needle from the embryo by jerking the needle to the side in a short and swift motion. Leave some Ringer's solution on either side of the cell column, and restrict the cells to the tapered end of the needle (Figure 1C,D).

NOTE: The liquid in front of the cell column will help to force apart the cells of the host embryos when depositing the cell column.

8. Clear any remaining yolk or cell debris by slowly moving the plunger up and down - while the needle remains immersed - to wash the cells with Ringer's solution. If done correctly, the undamaged cells 
will remain adhered together in a column while the debris is washed away.

NOTE: Great care must be taken during this step as the water level will rise past the tapered end of the needle. This will cause a sudden influx of water, which can be used to wash the cells. However, once the water level has passed the tapered end, the fine control with the plunger will be reduced, and the plunger has to be moved more slowly and carefully.

9. Move the transplantation dish with the non-dominant hand to position the needle orthogonal to the surface (either animal pole or margin) of the host embryo (Figure 1D-F).

10. Gently apply slight pressure, and then give a swift, sharp movement to pierce the enveloping layer of the host embryo. Take care not to scratch the yolk with the needle.

NOTE: Applying slight pressure onto the embryo by carefully squeezing it against the walls of the well increases the embryo's surface tension and thus facilitates the piercing of the enveloping layer.

11. Once the needle is inside, gently push the plunger to extrude the column of cells into the embryo while slowly retracting the needle at the same time (Figure 1D-F).

4. Cleaning the transplantation needle.

1. Rinse the needle by moving the plunger up and down while the needle is immersed in deionized water.

NOTE: If the needle remains dirty, rinse it with 10 M sodium hydroxide solution before rinsing it again with water.
2. Store the needle in an appropriate box for further usage.

\section{Generating ectopic sources of secreted signaling molecules in zebrafish embryos}

1. Preparing the host and donor embryos.

1. Collect freshly laid embryos by mating zebrafish.

2. Dechorionate 1-cell stage zebrafish embryos by incubating up to 100 embryos in $0.5 \mathrm{mg} / \mathrm{mL}$ of pronase solution for approximately $15 \mathrm{~min}$ in a small glass Petri dish (a detailed description of this procedure has been published by Rogers, K. W. et al. $\left.{ }^{14}\right)$.

NOTE: Alternatively, the embryos may also be dechorionated at the high stage just before transplantation (step 2.2), but this requires the injected donor embryos and uninjected host embryos to be dechorionated separately.

3. Submerge the embryos in embryo medium in a 200 $\mathrm{mL}$ beaker.

NOTE: Dechorionated blastula and gastrula stage embryos are very delicate. Their exposed yolk will adhere to plastic surfaces and rupture upon contact with air. Hence, they must remain completely immersed in embryo medium, transferred with a glass pipet, and maintained in either agarose-coated ( $1 \%$ in embryo medium) plastic dishes or glass Petri dishes.

4. Carefully empty out most of the embryo medium and slowly fill the beaker with fresh embryo medium. The mild agitation caused this way will facilitate the removal of the weakened chorions. Repeat this step 2-3 times. 
5. Transfer the dechorionated embryos using a glass Pasteur pipette into an agarose-coated injection dish.

NOTE: Flaming the tip of the glass Pasteur pipette by exposing it to a Bunsen burner flame can melt and smooth out the edge, helping to prevent damage to the embryos.

6. Inject the mRNA encoding the fluorescently tagged protein into a subset of embryos (a detailed description of this procedure has been published by Rogers, K. W. et al. ${ }^{14}$ ). Inject the mRNA into the cell, not the yolk, for near-homogeneous expression. Injected embryos will serve as donors, while uninjected embryos will serve as hosts.

NOTE: The amount and type of injected mRNA will depend on the signaling molecule being studied and usually ranges from 20 to $200 \mathrm{pg}^{2,4,5,6}$ (but can be as high as $1000 \mathrm{pg}$ in certain cases ${ }^{4}$ ).

7. Transfer the injected embryos to an agarose-coated six-well dish filled with embryo medium. Incubate at $28{ }^{\circ} \mathrm{C}$ until the embryos reach the early sphere stage.

2. Transplanting cells to generate ectopic sources.

1. Fill a transplantation dish (with individual triangular wedge-shaped wells, see Table of Materials) with Ringer's solution (116 mM NaCl, $2.8 \mathrm{mM} \mathrm{KCl}, 1 \mathrm{mM}$ $\mathrm{CaCl}_{2}, 5 \mathrm{mM}$ HEPES; store the HEPES buffer at 4 $\left.{ }^{\circ} \mathrm{C}\right)$.

NOTE: The calcium in Ringer's solution promotes cell adhesion and helps the embryo heal from the transplantation procedure.

2. Transfer the embryos into the transplantation dish.
3. Position the host and donor embryos in alternating columns, in each case with the animal pole oriented toward the transplantation needle.

4. Carry out the transplantation as described in section 1.3. For ectopic source transplantation, take source cells from the top of the animal pole and deposit them into the same location in the host embryo (Figure 1E).

NOTE: Washing the cells with Ringer's solution as detailed in step 1.3.8 is important for ectopic source generation to make sure that already secreted signaling molecules are not carried over to the host. Do not transplant too many cells to ensure that the source is not dispersed. A column approximately 80 $\mu \mathrm{m}$ in diameter and $100 \mu \mathrm{m}$ in length is appropriate for many applications.

5. Allow the host embryo to stay in Ringer's solution for $30 \mathrm{~min}$ to $1 \mathrm{~h}$ to recover.

6. Examine whether cells were successfully transplanted using a fluorescence stereomicroscope (Figure 2).

7. After recovery, transfer the embryos to an agarosecoated six-well plate filled with embryo medium and incubate them at $28^{\circ} \mathrm{C}$.

\section{Generating size-reduced embryos by cell extirpation}

1. Preparing embryos for extirpation.

1. Collect freshly laid embryos from zebrafish of the desired genotype.

2. Incubate the embryos at $28^{\circ} \mathrm{C}$ until they reach the high stage. 
3. Dechorionate the embryos as described in steps 2.1.2-2.1.5 when the embryos reach the high stage. NOTE: In this example, cell extirpation is performed at the sphere stage. Accordingly, embryos are dechorionated approximately $30 \mathrm{~min}$ to $1 \mathrm{~h}$ earlier.

2. Optional: Labeling the yolk syncytial layer (YSL).

NOTE: If desired, inject fluorescent dextrans into the YSL before extirpating cells. This technique allows to determine whether the YSL remains intact after cell removal, which is important for normal embryogenesis ${ }^{15}$. Alternatively, in vitro synthesized mRNAs or proteins can also be injected into the YSL.

1. Use a Pasteur pipette to transfer the dechorionated embryos to an agarose-coated injection dish filled with embryo water.

2. Orient the embryos laterally, with the blastoderm margin pointing toward the injection needle (Figure 3A).

3. Inject $0.5 \mathrm{~nL}$ of $1.5 \mu \mathrm{g} / \mu \mathrm{L} 10 \mathrm{kDa}$ Alexa568Dextran into the YSL, aiming for a region between the blastoderm cells and the yolk at a depth of about one-third of the embryo's diameter. Inject all embryos within one row.

NOTE: Since the injection needle does not need to pierce through the chorion, a small $(\sim 4 \mu \mathrm{m})$ and sharp opening is advantageous.

4. Rotate the embryos by $180^{\circ}$ so that the opposite side of the blastoderm margin is pointing toward the injection needle (Figure 3A).

5. Inject another $0.5 \mathrm{~nL}$ of $1.5 \mu \mathrm{g} / \mu \mathrm{L} 10 \mathrm{kDa}$ Alexa568Dextran into the YSL as described in step 3.2.3, yielding a total injection volume of $1 \mathrm{~nL}$ per embryo.
NOTE: Injecting from two sides allows for a more even distribution of fluorescent dextran within the YSL.

6. Examine the result of the injection under a fluorescence stereomicroscope. If done correctly, the fluorescent signal will be restricted to the YSL (Figure 3B) and not be visible in the intercellular space of the blastoderm.

3. Extirpating cells

1. Fill a transplantation dish with Ringer's solution (see step 2.2.1).

2. Transfer the embryos to the transplantation dish.

3. Orient the embryos with the animal pole toward the transplantation needle (Figure 3C).

4. Carefully remove cells from the animal pole region as described in steps 1.3.5-1.3.7, thereby reducing the blastoderm to the desired size.

5. Discard the removed cells by expelling them from the transplantation needle.

6. Once the procedure is completed, allow the embryos to stay in Ringer's solution for $30 \mathrm{~min}$ to $1 \mathrm{~h}$ to recover.

7. OPTIONAL: Examine the integrity of the YSL under a fluorescence stereomicroscope (Figure 3D).

8. Transfer the embryos to an agarose-coated plate filled with embryo medium and incubate them at 28 ${ }^{\circ} \mathrm{C}$.

\section{Creating maternal-zygotic mutants by germline transplantation}

1. Preparing host and donor embryos. 
1. Collect freshly laid embryos from both mutant and wild-type zebrafish. Embryos from zebrafish with a mutant background will serve as donors, while embryos from zebrafish with a wild-type background will serve as hosts.

NOTE: Germline transplantation requires a high number of starting embryos ( 50 for donors, $~ 500$ for hosts) to ensure a good number of successful germline transplants that survive into adulthood.

2. Transfer the embryos to an agarose-coated injection dish with embryo medium using a Pasteur pipette. NOTE: Injections are done before dechorionation to increase throughput. Needles for injection through the chorion need to be blunt and have a larger opening $(\sim 10 \mu \mathrm{m})$ to prevent clogging.

3. Inject the donor embryos with $1 \mathrm{~nL}$ of $100 \mathrm{ng} / \mathrm{\mu L}$ mRNA encoding GFP with a nos1 3'UTR. Inject the mRNA into the yolk to increase the throughput.

NOTE: The nos1 3'UTR will stabilize the mRNA in the primordial germ cells, causing the germ cells to be strongly fluorescent when imaged 1 day postfertilization $^{10}$.

4. Inject the host embryos with $1 \mathrm{~nL}$ of $0.33 \mathrm{mM}$ (3 $\mu \mathrm{g} / \mu \mathrm{L})$ dead end (dnd) morpholino. Inject the morpholino into the yolk to increase the throughput. NOTE: The dnd morpholino blocks primordial germ cell formation, which ensures that the host embryo's germline will be exclusively populated with cells from the donor after transplantation ${ }^{10}$.

5. Transfer the injected embryos to plastic Petri dishes with embryo medium. Incubate at $28^{\circ} \mathrm{C}$ until the embryos reach the high stage.
6. Dechorionate the embryos as described in steps 2.1.2-2.1.5 when the embryos reach the high stage.

2. Transplantation of germ cells

1. Fill a transplantation dish with Ringer's solution (see step 2.2.1).

2. Transfer the dechorionated sphere- to dome-stage embryos into the transplantation dish.

3. Position the host embryos and donor embryos in alternating columns to transplant the cells from one donor embryo to six different host embryos. This increases the chance that germ cells from a given host embryo will successfully be transplanted.

4. Orient the embryos with the margin toward the transplantation needle and carry out the transplantation as described in section 1.3. For germline transplantations (Figure 1D,F), take the source cells from the margin (where the primordial germ cells are located) and deposit them into the same location in the host embryo.

NOTE: Transplanting a large column (approximately $80 \mu \mathrm{m}$ in diameter and $600 \mu \mathrm{m}$ in length) will increase the chance of obtaining a successful germline transplant.

5. Allow the embryo to stay in Ringer's solution for 30 $\min$ to $1 \mathrm{~h}$ to recover once the transplantation is completed.

NOTE: If not all donor embryos are expected to be homozygous mutants - e.g., if they result from a heterozygous incross - they need to be genotyped after transplantation to determine the genotype of the host's transplanted future germline. In this case, ensure that the positions of the host and donor embryos are not mixed up during handling. 
6. Use a fluorescence stereomicroscope to examine whether the cells were successfully transplanted (Figure 4A,B).

7. Transfer the embryos into a 24-well agarose-coated plate. Group all the host embryos that received cells from the same donor into the same well and label them accordingly. Incubate them until the next day at $28^{\circ} \mathrm{C}$

8. If required, transfer the donor embryos into labeled PCR strips for genotyping.

3. Screening for successful germline transplants

1. Screen the host embryos for successful germline transplants under a fluorescence stereomicroscope approximately $30 \mathrm{~h}$ post-fertilization (hpf). The germ cells are found at the groove above the yolk extension (Figure 4C).

NOTE: Typical experiments with the device and strategy presented here will have a success rate of $\sim 60 \%-80 \%$ to obtain at least one host embryo carrying transplanted germ cells per donor embryo. A previous report described a $\sim 10 \%$ efficiency ${ }^{10}$.

2. If applicable, discard embryos that received cells from donor embryos with an incorrect genotype. Grow larvae with successfully transplanted germ cells to adulthood according to standard husbandry conditions and following institutional guidelines.

\section{Representative Results}

Success and failure in the usage of the transplantation device for the three applications described above can readily be assessed by visual inspection under a stereomicroscope. In successful transplantations, the embryo should look normal and similar in shape and yolk clarity to untransplanted embryos, without large tears in the blastoderm. If the embryo is visibly damaged (Figure 4B), it will not develop normally. Ideally, transplanted cells expressing a fluorescent marker should appear as a continuous column when viewed under a fluorescence stereomicroscope (Figure 2A, Figure 4A). If the column is fragmented, this indicates that the cells were sheared by the suction into the transplantation needle or that the deposition of the cells was done too vigorously. This can be prevented by moving the plunger more slowly and gently.

Although the transplantation device has mainly been used on zebrafish embryos at blastula stages ${ }^{5,6}$, transplantation and cell extirpation work just as well for dechorionated blastulastage embryos of the Japanese rice fish medaka (Oryzias latipes) (Figure 2B). Apart from embryo dechorionation, which has been described by Porazinski, S. R. et al. ${ }^{17}$, the same procedures as described above can be followed.

In the specific case of germline transplantation, a good transplant will result in an embryo with a long horizontal column of cells directly above the yolk margin (Figure 4A). However, whether germ cells were successfully transplanted can only be assessed on the following day (Figure 4C-F) due to background expression of GFP at blastula stages (Figure 1F). The primordial germ cells will appear as small fluorescent spheres in the groove directly above the yolk extension (Figure 4C-E). The presence of these cells at the correct location indicates successful germline transplantation. Cells with a different shape are not germ cells (e.g., elongated cells are typically muscle cells, Figure 4F). Also, if primordial germ cells are found outside the groove, this means that they have failed to migrate properly, and they will not be able to contribute to the embryo's germline. Finally, the general morphology of transplanted embryos should appear similar to untransplanted embryos (Figure 4D); the tail should 
not be deformed, and the head should not be shrunken or missing eyes (Figure 4E). These defects generally result from excessively high morpholino concentrations or from embryo damage during transplantation. Germline transplantation experiments as described here will typically result in 1-2 out of
6 host embryos with successfully transplanted germ cells for $60 \%-80 \%$ of donor embryos, depending on the experience of the experimenter. Thus, cells from 40-50 homozygous donor embryos need to be transplanted into $200-300$ host embryos to raise approximately 30 individuals with mutant germ cells. 

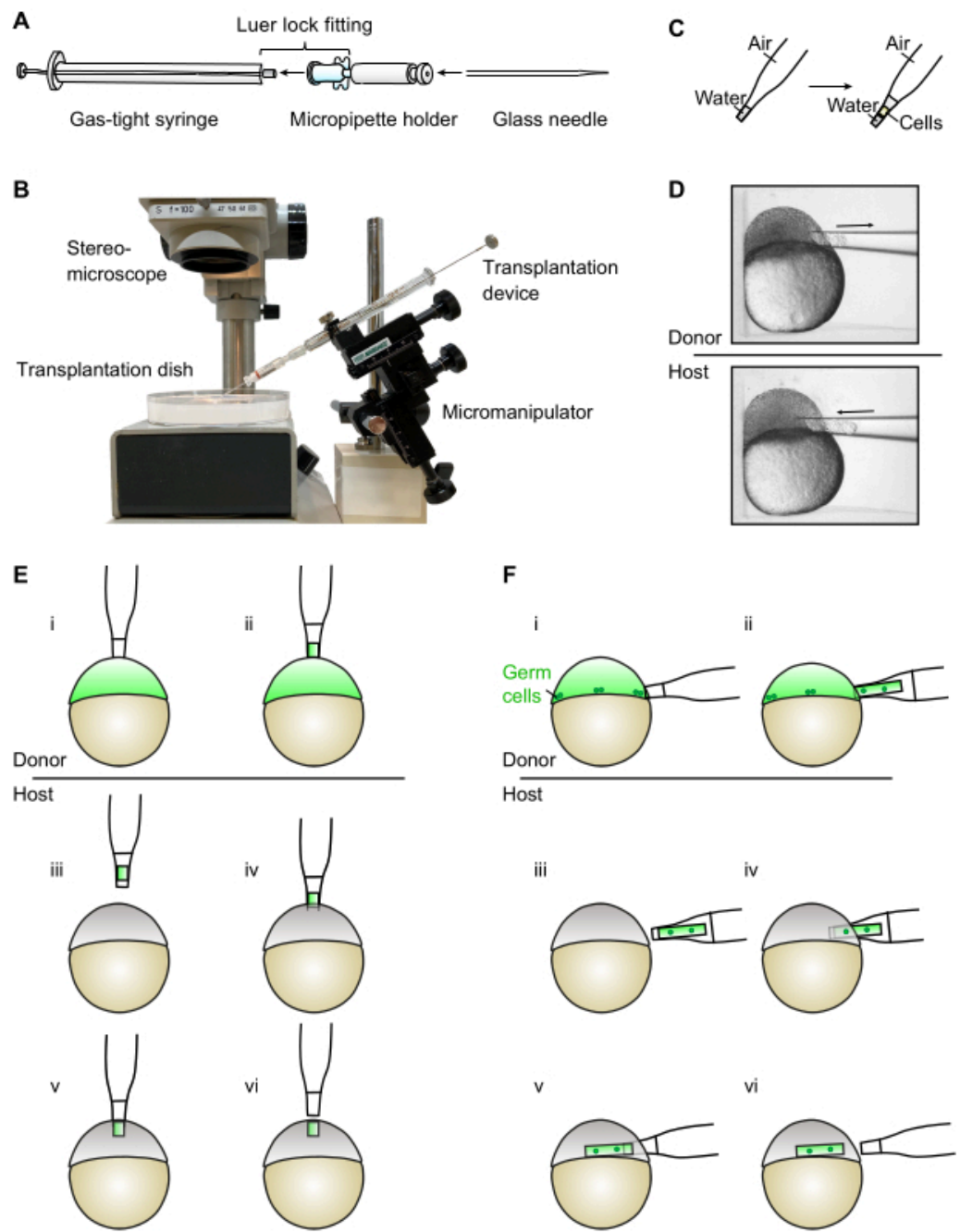

Figure 1: Assembly and usage of the transplantation device. (A) The transplantation device is assembled by connecting a gas-tight syringe with a micropipette holder through Luer lock fitting. The glass needle for transplantation is then inserted into the micropipette holder. (B) Photograph of the assembled transplantation device mounted onto a micromanipulator (please note that background and labels have been removed from the picture). (C) When using the transplantation device, it is important to ensure that the water level in the transplantation needle remains at the tapered end. (D) The device is used under a stereomicroscope to withdraw and insert cells from and into teleost embryos placed in individual wells of a transplantation dish. (E) For the generation of ectopic sources, cells are taken from the animal pole of a donor embryo (iii) and transferred into the animal pole of a host embryo (iii-vi). (F) For germline transplantation, a larger number of cells is taken from the margin of a donor embryo (i-ii), where the germ cells are located. The cells are then transferred into the margin of host embryos (iii-vi). Please click here to view a larger version of this figure. 

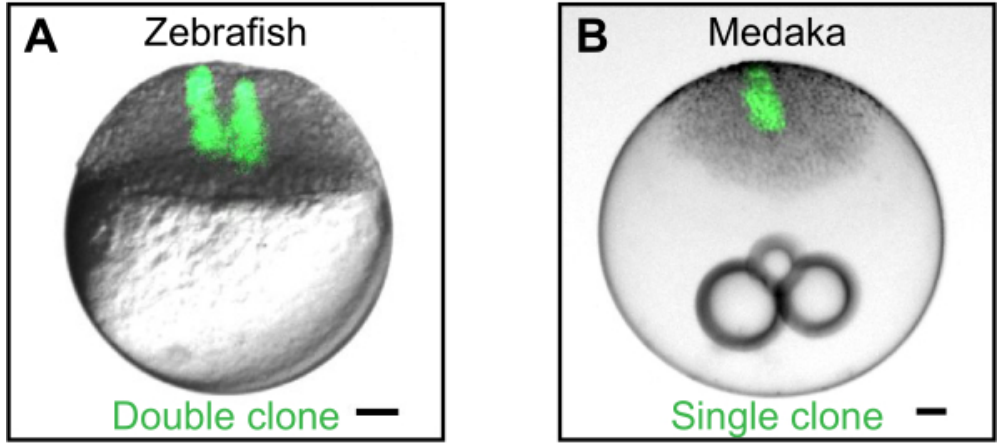

Figure 2: Generating clones by cell transplantation. (A) Example of a double clone generated by sequential transplantation of fluorescent cells (green) from a zebrafish donor into a zebrafish host embryo. Single and double clones can be used to study how secreted signaling molecules form spatiotemporal gradients ${ }^{2,4,5,6}$. (B) Example of a single clone generated by transplanting cells from a transgenic eGFP-expressing medaka donor (Wimbledon $)^{17}$ into a wild-type medaka host. Scale bars represent $100 \mu \mathrm{m}$. Please click here to view a larger version of this figure. 

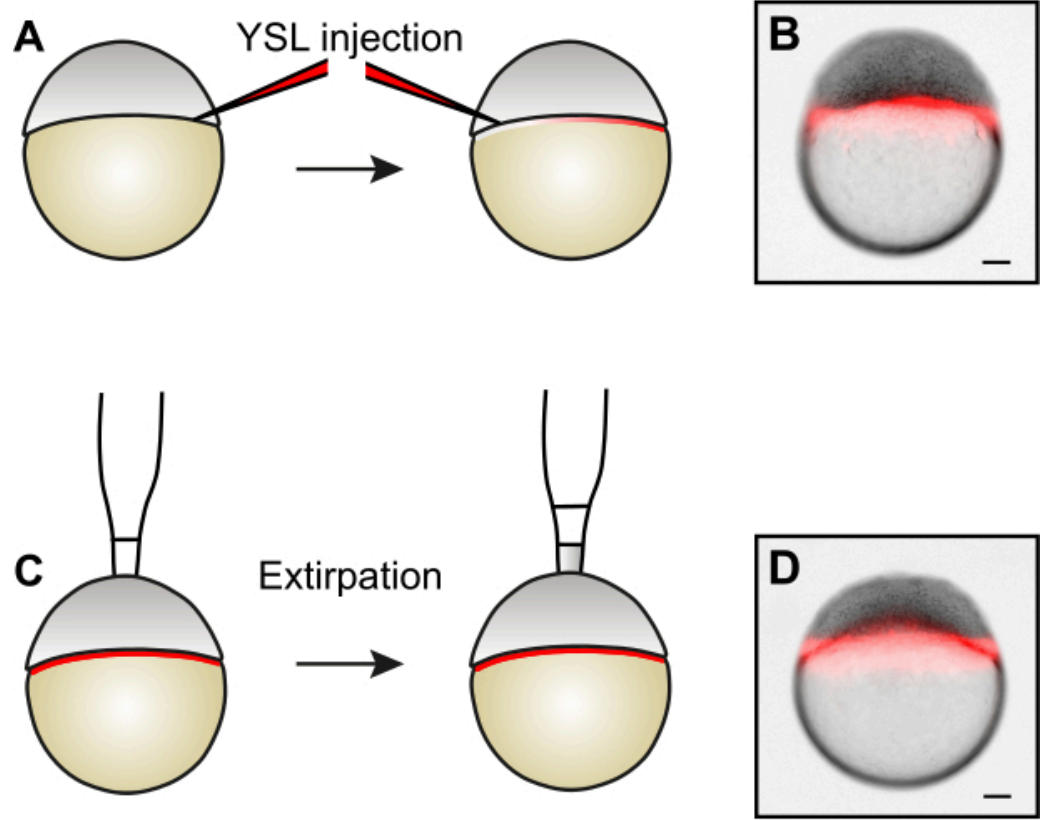

Figure 3: Generating size-reduced embryos by cell extirpation. (A) Before removing cells by extirpation, the YSL can be labeled by injecting fluorescent dyes into two opposing sides of the YSL. (B) Example of an embryo after YSL injection. (C) To generate size-reduced embryos, cells from the animal pole are removed by extirpation ${ }^{5}$. (D) Example of an embryo after cell extirpation. Note that the YSL stays intact. Scale bars represent $100 \mu \mathrm{m}$. Please click here to view a larger version of this figure. 

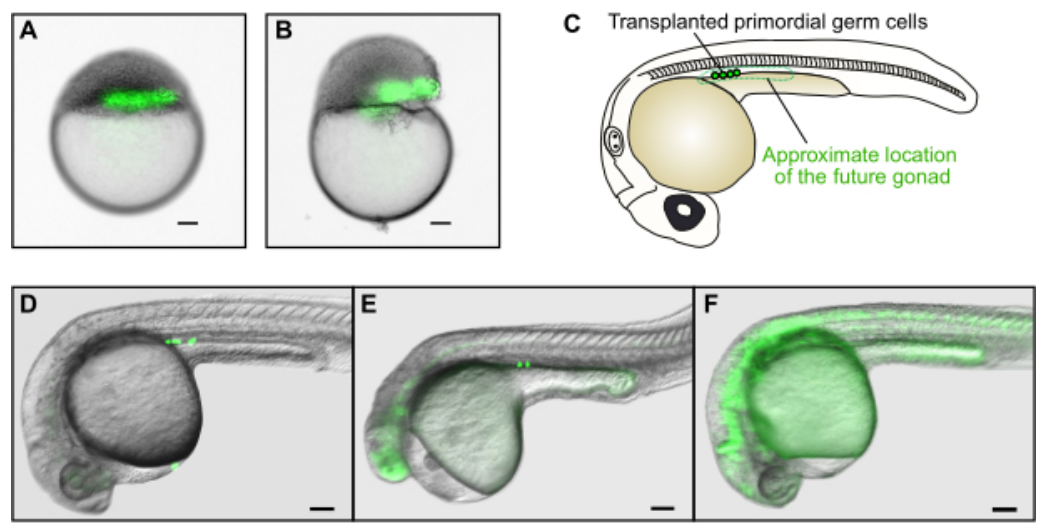

Figure 4: Germline transplantation. (A) Example of a successful transplantation of donor cells (green) into the host's marginal zone. (B) Example of an unsuccessful transplantation. The yolk of the host embryo was severely damaged, and the embryo will not be able to develop normally. (C) At $30 \mathrm{hpf}$, successfully transplanted germ cells will be found solely in the gonadal mesoderm at the anterior region of the yolk extension. (D) Example of a successful transplantation in which several GFP-labeled donor germ cells have populated the host's future gonad. (E) Example of an unsuccessful transplantation. Although germ cells have reached the gonadal mesoderm, the host embryo is severely deformed and will not develop normally. (F) Example of an unsuccessful transplantation. Fluorescent germ cells that failed to migrate into the correct location will not repopulate the gonads. Scale bars represent $100 \mu \mathrm{m}$. Images in D-F were taken at a total magnification of approximately 50x. Please click here to view a larger version of this figure.

\section{Discussion}

The success of a transplantation experiment strongly relies on the fine motor skills of the experimenter. To successfully carry out the procedures, practice is required. However, the instrument presented here is relatively easy to learn and use compared to others on the market, and, in general, only a few days of practice are needed.

The success of the transplantation procedure can be enhanced by taking several precautions. One step is to ensure that the micromanipulator is of good quality and capable of smooth operation. Adding an ocular with higher magnification to the stereomicroscope can help to precisely position the needle relative to the embryo. Using well- breeding zebrafish or medaka to acquire healthy embryos and taking care not to damage the embryos during handling (especially during and after the dechorionation step) will also enhance the success rate.

Problems with delayed toxicity can be more difficult to troubleshoot. If an embryo dies after a few hours - but not immediately following transplantation - the yolk might have been damaged by the needle (e.g., by entering the embryo too deeply), or perhaps the cells were ejected too forcefully. Delayed toxicity and embryonic death can also result from yolk or cell debris injected along with the donor cells; another cause can be deteriorating HEPES buffer in the Ringer's solution. These problems can be overcome by washing the 
cells (see step 1.3.8) or by simply using a fresh batch of buffer, respectively. Furthermore, deformed host embryos in germline transplantation experiments might result from excessively high morpholino concentrations. It is crucial to use enough morpholino to fully ablate the host's wild-type germline, thereby preventing these cells from contributing to the offspring - but at the same time, excessively high morpholino concentrations need to be avoided. Consistent morpholino amounts across all injected host embryos (a few hundred in a typical experiment) are therefore key to the success of germline transplantations. This can be helped by supplementing the morpholino injection mix with an easily visible tracer dye ${ }^{14}$, which can be tracked under a fluorescence stereomicroscope to ensure that all embryos receive the same injection volume.

The procedures described in this protocol exclusively involve manipulations of cells in blastula-stage zebrafish or medaka embryos, but in the future, it will likely be possible to adapt the device to different stages and species by changing the diameter and shape of the transplantation needle.

\section{Disclosures}

The authors have no conflicts of interest to declare.

\section{Acknowledgments}

This project was supported by the Max Planck Society and received funding from the European Research Council (ERC) under the European Union's Horizon 2020 research and innovation program (grant agreement No. 637840 (QUANTPATTERN) and grant agreement No. 863952 (ACEOF-SPACE)).

\section{References}

1. Spemann, H., Mangold, H. Induction of embryonic primordia by implantation of organizers from a different species. Archives for Microscopic Anatomy and Developmental Mechanics. 100 (3-4), 599-638 (1924).

2. Müller, P. et al. Differential diffusivity of Nodal and Lefty underlies a reaction-diffusion patterning system. Science. 336 (6082), 721-724 (2012).

3. Donovan, P. et al. Paracrine Activin-A signaling promotes melanoma growth and metastasis through immune evasion. Journal of Investigative Dermatology. 137 (12), 2578-2587 (2017).

4. Pomreinke, A. P. et al. Dynamics of BMP signaling and distribution during zebrafish dorsal-ventral patterning. eLife. 6, 25861 (2017).

5. Almuedo-Castillo, M. et al. Scale-invariant patterning by size-dependent inhibition of Nodal signaling. Nature Cell Biology. 20, 1032-1042 (2018).

6. Soh, G. H., Pomreinke, A. P., Müller, P. Integration of Nodal and BMP signaling by mutual signaling effector antagonism. Cell Reports. 31 (1), 107487 (2020).

7. Mahalwar, P., Walderich, B., Singh, A. P., NüssleinVolhard, C. Local reorganization of xanthophores finetunes and colors the striped pattern of zebrafish. Science. 345 (6202), 1362-1364 (2014).

8. Frohnhöfer, H. G., Krauss, J., Maischein, H. M., NüssleinVolhard, C. Iridophores and their interactions with other chromatophores are required for stripe formation in zebrafish. Development. 140 (14), 2997-3007 (2013).

9. Chen, Y., Schier, A. F. The zebrafish Nodal signal Squint functions as a morphogen. Nature. 411 (6837), 607-610 (2001). 
10. Ciruna, B. et al. Production of maternal-zygotic mutant zebrafish by germline replacement. Proceedings of the National Academy of Sciences of the United States of America. 99 (23), 14919-14924 (2002).

11. Westerfield, M. The zebrafish book. A guide for the laboratory use of zebrafish (Danio rerio). 4th edn. University of Oregon Press (2000).

12. Kemp, H. A., Carmany-Rampey, A., Moens, C. Generating chimeric zebrafish embryos by transplantation. Journal of Visualized Experiments:JoVE. 29, 1394 (2009).

13. apek, D., Müller, P. Positional information and tissue scaling during development and regeneration. Development. 146 (24), dev177709 (2019).

14. Rogers, K. W., Bläßle, A., Schier, A. F., Müller, P. Measuring protein stability in living zebrafish embryos using fluorescence decay after photoconversion (FDAP). Journal of Visualized Experiments: JoVE. 95, 52266 (2015).

15. Carvalho, L., Heisenberg, C. P. The yolk syncytial layer in early zebrafish development. Trends in Cell Biology. 20 (10), 586-592 (2010).

16. Porazinski, S. R., Wang, H., Furutani-Seiki, M. Dechorionation of medaka embryos and cell transplantation for the generation of chimeras. Journal of Visualized Experiments: JoVE. 46, 2055 (2010).

17. Centanin, L., Hoeckendorf, B., Wittbrodt, J. Fate restriction and multipotency in retinal stem cells. Cell Stem Cell. 9 (6), 553-562 (2011). 